\title{
Protocol Deviations: A Holistic Approach from Defining to Reporting
}

\author{
Laura Galuchie ${ }^{1}\left(\mathbb{D} \cdot\right.$ Catherine Stewart, $\mathrm{PhD}^{2} \cdot$ Frank Meloni, $\mathrm{PhD}^{3}$
}

Received: 30 October 2020 / Accepted: 25 February 2021 / Published online: 29 March 2021

(c) The Author(s) 2021

\begin{abstract}
Improving interpretation of existing guidelines and management of protocol deviation processes could increase process efficiencies and help reduce noise to support rapid identification of important protocol deviations. Towards this end, TransCelerate identified key principles to build upon and clarify the definition of a protocol deviation and developed a holistic approach to protocol deviation management. The approaches are flexible to suit a variety of indications, study designs, and investigational agents while also supporting consistent application within a study, program or organization.
\end{abstract}

Keywords Protocol deviation (PD) · Quality Management System (QMS) · Risk management $\cdot$ Issue management · Risk assessment categorization tool (RACT) · TransCelerate

\section{Introduction}

Clinical study protocols are conducted according to the International Council for Harmonization guidance on Good Clinical Practice (GCP) [1] which outlines safeguards for the rights, safety and well-being of participants. Protocols "should [also] be designed, conducted and analyzed according to sound scientific principles to achieve their objectives; and should be reported appropriately" [2] If conducted as designed, the data produced should be reliable and reproducible, supporting a clear interpretation of results while protecting participants. It seems intuitive that deviations to the protocol could negatively impact the participant or interpretability of the data and should be avoided.

Nonetheless, despite efforts to minimize them, protocol deviations (PDs) do occur. They do not all have the same impact and the importance of the deviation needs to be assessed. Examples of important PDs, defined as those with the most impact, were provided in ICH E3 in 1996 [3]. A

Laura Galuchie

laura.galuchie@merck.com

1 Global Clinical Trial Operations, Merck Research Laboratories, 126 East Lincoln Ave, Rahway, NJ 07065, USA

2 Clinical Development \& Operations, Pfizer R\&D UK Ltd, Sandwich, Kent, UK

3 Regulatory Medical Writing, Janssen Research \& Development, Spring House, PA, USA formal definition and additional examples were provided in ICH E3 Q\&A R1 in 2012 [4]. Despite a formal definition with examples, different interpretations of importance exist $[5,6]$.

Over-interpretation of this definition may lead to the inclusion of situations which are not PDs, such as theoretical situations. The addition of these extraneous situations could potentially delay identification of important patient safety information by increasing noise in the system. Underinterpretation may exclude situations based on fault or other reasons and could decrease the reliability of study results related to both effectiveness and safety. This range of interpretation contributes to varied and sometimes conflicting instruction to sites. This limits their ability to identify PDs and establish preventative actions which may result in direct impact to participants. It may also delay reporting or obscure interpretation of PDs by institutional review boards (IRBs) or ethics committees (ECs).

\section{Methods}

Subject matter experts from TransCelerate Member Companies formed a cross-functional working group to understand common challenges related to PDs. To confirm and further articulate issues, the team conducted blind surveys and interviews and reviewed literature and multiple guidelines. A common factor seen across stakeholders was variation in the definition of important PDs. In response, the team developed 
key principles building upon existing definition and incorporated ICH E6 R2 elements and associated methodology. Health authority comments and questions were addressed, resulting in a toolkit that was posted for public comment. Industry representatives were informed of a public comment period via conference presentations [7-9] and webinars [10, 11]. Public comments were incorporated into the toolkit.

\section{Results and discussion}

Key principles were developed to build upon and clarify the definition of a PD. However, applying a refined PD definition is not a one-time effort. To guide sponsors, CROs and investigational sites in identification and management of PDs, a toolkit was developed. It incorporates risk-based approaches from ICH E6 R2 and risk and issue management concepts [12] from the TransCelerate clinical Quality Management System conceptual framework [13]. The toolkit components are designed to be used together, but the approaches are flexible to meet the needs of study designs and stakeholders. Examples provided are not intended to be all-inclusive, exhaustive, or mandatory.

The Protocol Deviation Process Map (Fig. 1) illustrates a holistic approach to management of PDs. Process steps are repeated throughout the clinical study as emphasized by the feedback loops and ongoing activities, which are illustrated as horizontal bars.

\section{Define}

ICH E3 Q\&A R1 defines a PD as “....any change, divergence, or departure from the study design or procedures defined in the protocol." This definition is often over-interpreted leading to inclusion of a wide scope of items such as theoretical situations, and situations which are not PDs. For example, discovery that training of a Clinical Research Associate (CRA) was delayed needs to be addressed, but it is not a PD. This wide scope of items generates noise and could delay identification of trends or dilute impact of actual PDs.

For these reasons, we recommend the following clarifying key principles:

(1) An event occurred (e.g., not theoretical)

(2) The event is related to the protocol or documents referenced in the protocol (e.g., laboratory manual)

(3) The event is independent of fault, blame or circumstance - to ensure an objective approach to identification (e.g., sample tube broke en route to central laboratory)

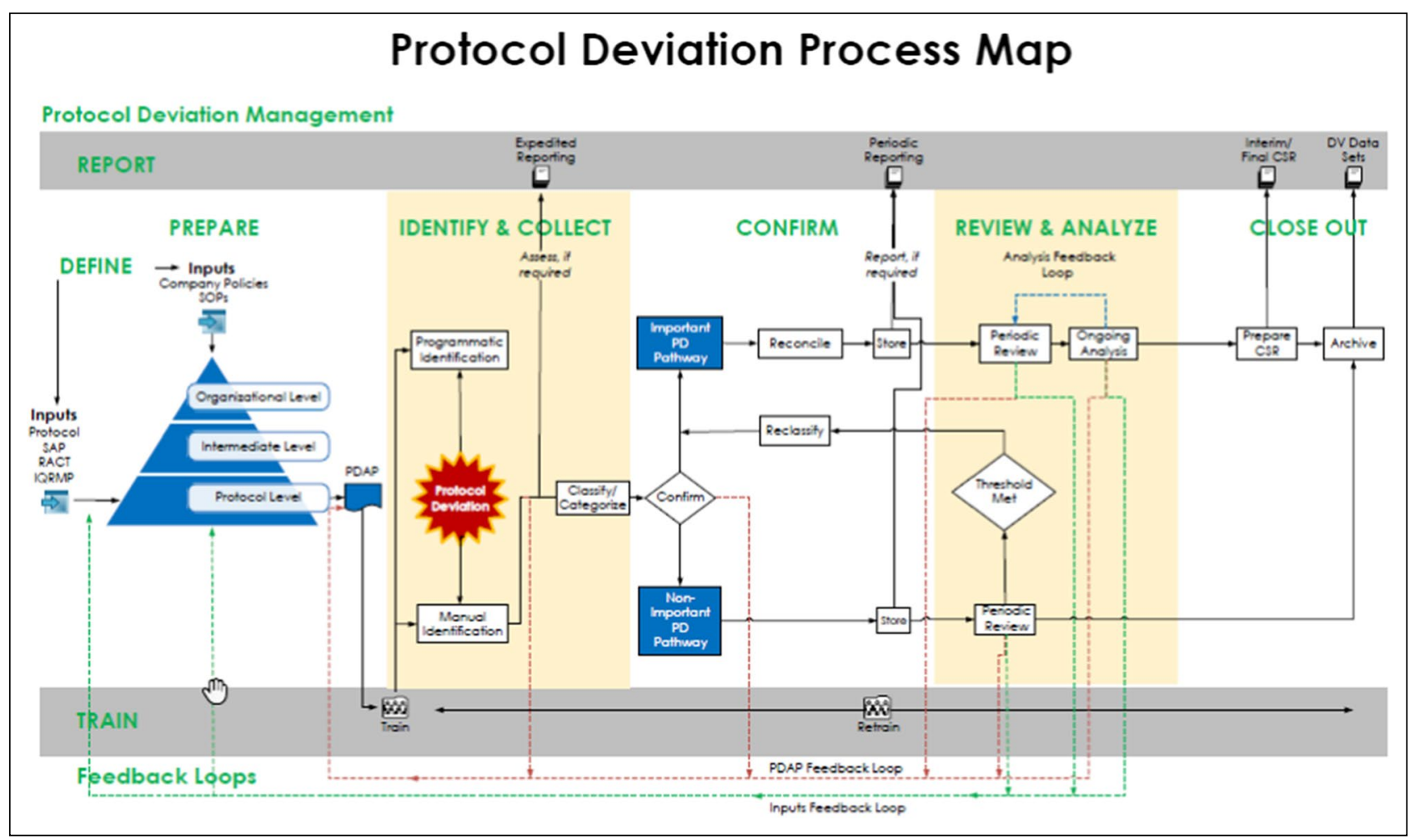

Fig. 1 Protocol deviation map 
Events, issues or situations that are not PDs may require action or follow-up through other processes.

Once a PD is identified, it can then be categorized as important or non-important. Risk-based approaches from ICH E6 R2 can be applied to the definition of important PDs. The updated definition becomes:

"Important protocol deviations are a subset of protocol deviations that may significantly impact the completeness, accuracy, and/or reliability of key study data or that may significantly affect a subject's rights, safety, or well-being. For example, important PDs may include enrolling subjects in violation of key eligibility criteria or failing to collect data necessary to interpret primary endpoints, as this may compromise the scientific value of the trial."

ICH E3 Q\&A R1 indicates sponsors have some flexibility in determining what is an important $\mathrm{PD}$, stating the "definition of important PDs for a particular trial is determined in part by study design, the critical procedures, study data, subject protections described in the protocol, and the planned analyses of study data." Building on this guidance we suggest the following interpretations:

- The term "protocol deviation" is preferred over the term "protocol violation." Local HA and IRB/EC may have other specific definitions;

- "Significant" in the context of PDs is not a statistical term;

- "Important," "major," "critical" and "significant" are synonyms when referring to important PDs.

Moving forward, use of "important" is proposed as common terminology.

The concepts of key or critical study data and processes are not new. They were outlined in the $2011 \mathrm{draft}$ and 2013 issuance of FDA's Guidance for Industry "Oversight of Clinical Investigators - A Risk-Based Approach Monitoring" [14] as well as the 2016 ICH E6 R2. They continue to be key component of risk-based approaches to clinical study management [15]. We believe the same risk-based principles apply to defining important PDs.

ICH E3 does not provide a formal definition of a nonimportant PD. It appears reasonable, however, to consider a PD non-important if it does not meet the criteria for important. Both important and non-important PDs are collected, processed and reported, although the pathways may differ as discussed later.

The PD Decision Tree (Fig. 2) can be applied to support consistent application of critical thinking within an organization, and potentially across the industry. Conducting periodic therapeutic area or indication reviews is a best practice to maintain consistency within a company.

\section{GCP Compliance}

Clinical studies are conducted according to GCP. Protocols make direct reference to GCP, and some have assumed all GCP compliance issues are also PDs, thus inflating the volume of events. For example, a missing signature on the Delegation of Authority log needs to be addressed, perhaps as an action item. In most cases, this granular procedure is not written in the protocol and is not a PD.

To reduce noise generated by these types of events, we propose to address GCP issues outside the PD process unless they meet the classification of important as outlined via the PD Decision Tree (Fig. 2).

Examples of GCP compliance issues which may also be important PDs include:

- Study participant received expired investigational product

- Key or critical study procedures performed by study site staff without appropriate qualifications or training

Some GCP compliance issues may also qualify for expedited reporting to Regulatory Authorities depending on local regulatory requirements (e.g., serious breaches). Companies should follow their escalation and assessment paths for decision making and reporting.

\section{Protocol}

The protocol and documents referenced in the protocol are the primary sources when determining whether something is or is not a PD. Therefore, a best practice is to conduct risk assessment reviews and define PD classification approaches prior to protocol finalization. This allows for changes to reduce occurrence of PDs.

\section{Prepare}

A protocol-specific protocol deviation assessment plan (PDAP) documenting the management of PDs is recommended to support consistency within a study, across a program, and within a company or organization. As illustrated in Fig. 3, it may be created as a stand-alone document or incorporated into existing quality and risk management plans. Whatever the form, we recommend creation in conjunction with protocol development and maintenance as a living document, with suitable version control measures, until the last study data has been reviewed.

The first step is to define and prospectively identify important PDs which may occur. Inputs may come from organizational, intermediate and protocol level components as illustrated in Fig. 4. Although all three inputs are used, we recommend use of organizational and intermediate level 


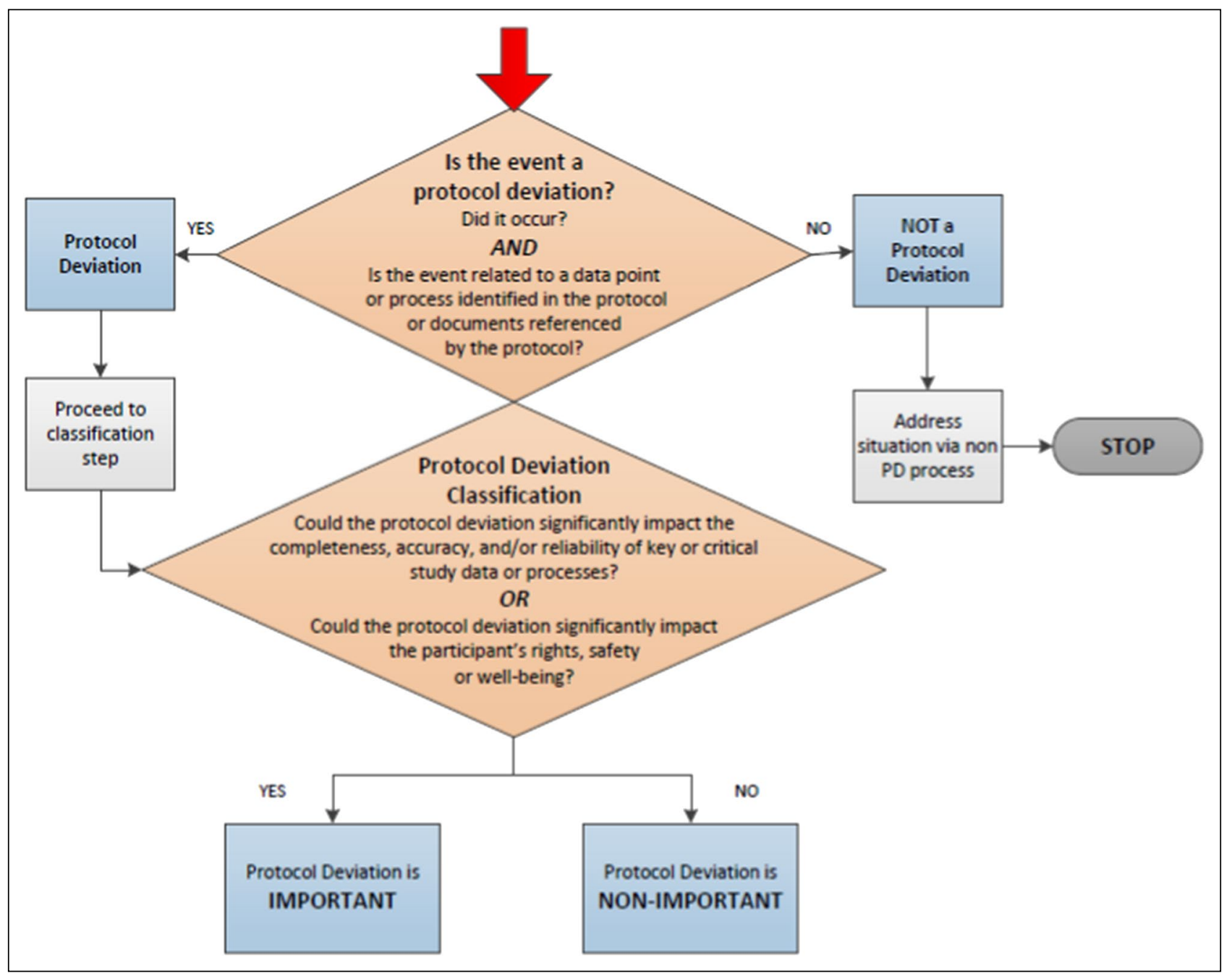

Fig. 2 Protocol deviation decision tree.

definitions whenever possible, augmented with protocol level definitions where necessary. This will reduce variability in classification and categorization and help support consistency in analysis and reporting.

Use of an issue management approach supports consistent identification, classification and categorization of PDs (Fig. 5) and consistent responses to the question "What is an important PD?".

Finally, teams may find program-level or protocol level risk assessment tools useful to identify potential situations which would be considered important PDs.

Under this proposed approach, the PDAP should describe PDs classified as important. A pragmatic approach should be applied when describing non-important PDs, for example, only include those commonly misclassified or part of a classification threshold.

The PDAP template includes the following recommended elements:
- Guidance for consistent classification and categorization of PDs,

- Method of identification for potential important PDs and primary team members involved,

- Thresholds at which non-important PDs may become important.

Additional elements may be included:

- Frequency of data reviews or trend analyses,

- Feedback and/or escalation pathways,

- Type and extent of reconciliation (e.g., between PD collection tool and the clinical database),

- Documentation, approval, and archiving requirements.

The PDAP is a living document and is intended to be reviewed and updated throughout the study. It should remain a living document until the last study data has been 


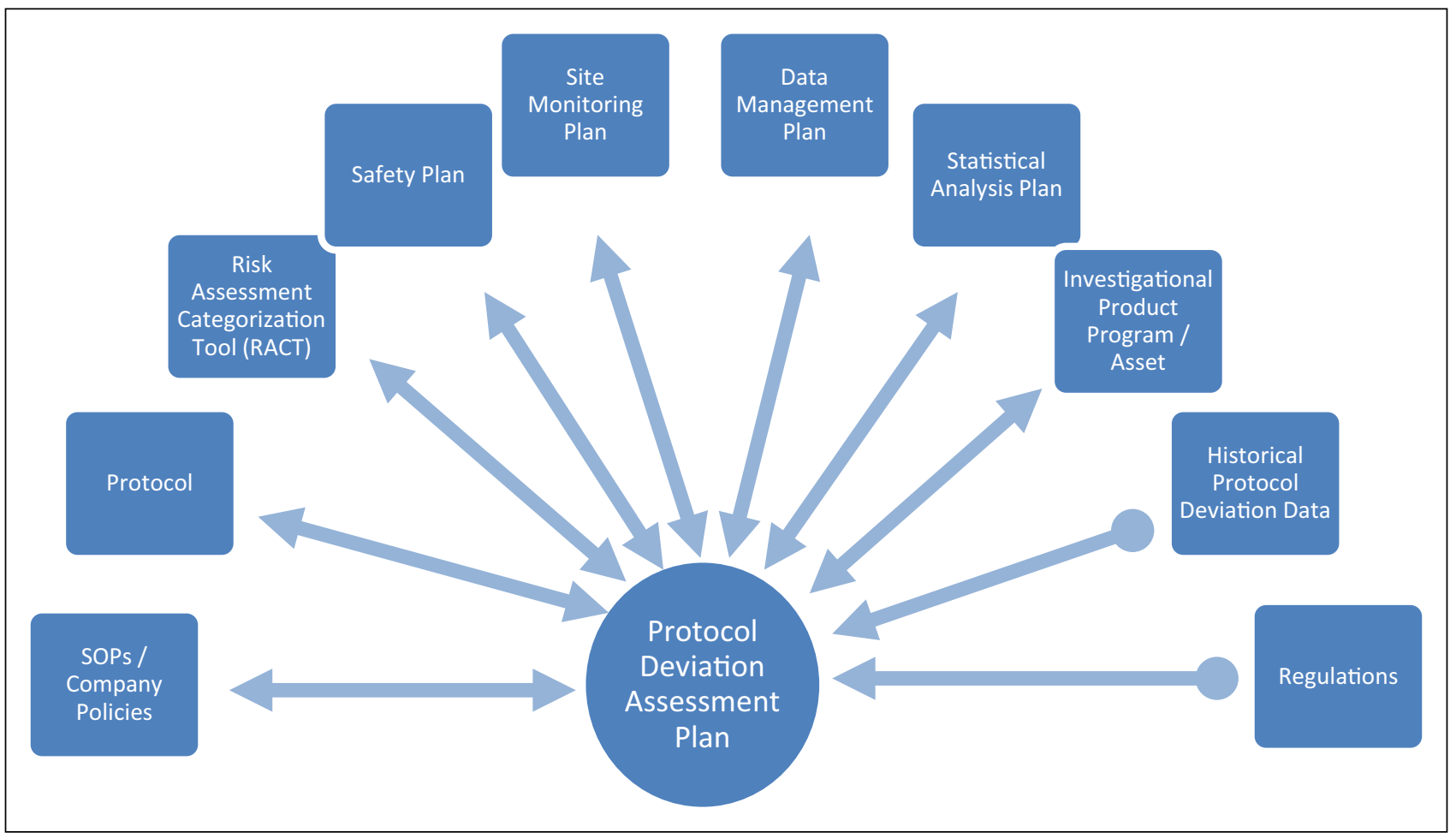

Fig. 3 Relationship between protocol deviation assessment plan (PDAP), quality and risk management plans and other documents

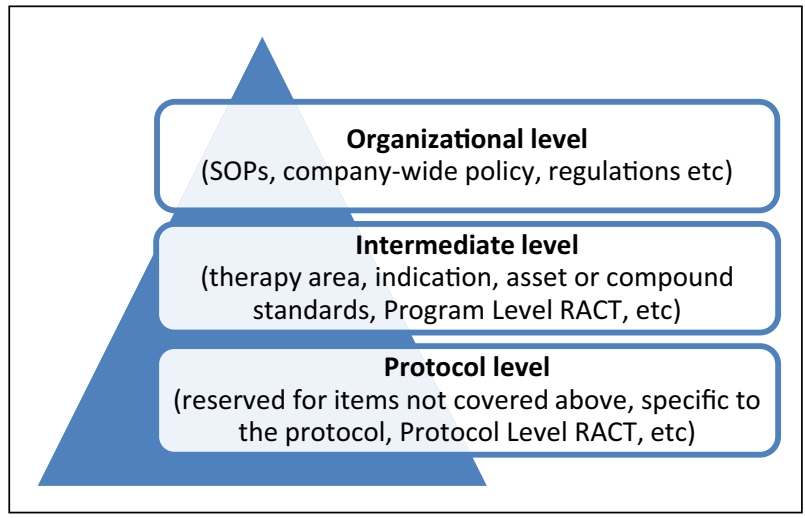

Fig. 4 PDAP levels: organizational, intermediate, protocol

reviewed. The PD Decision Tree (Fig. 2) can also be used during study conduct.

\section{Train}

Training should be provided to relevant study team members, emphasizing their role. As a best practice, the following approach is recommended:

- Train study site personnel on the protocol including amendments, not the PDAP,
- Train study team (including CRAs, CROs, vendor, etc.) on the protocol including amendments and the PDAP including updates.

Retraining and feedback mechanisms should also be implemented.

\section{Identify and Collect}

PDs can be identified via programmatic or manual processes. Programmatic identification is based on data captured in a database using an electronic/computerized process or program. Manual identification relies on human interpretation. In both cases, a well-defined PDAP is essential for consistency and timely identification of PDs. Describing the method of identification of potential important PDs ensures alignment of efforts and focuses team members on items which cannot be identified via the alternate approach.

Manual identification approaches can vary but should focus on information not captured in an electronic system. As a best practice, identification of potential PDs should rely on programming and reports whenever possible. The identification of those elements which are not programmable should be a primary focus for CRAs during on-site visits.

Application of risk-based monitoring methodology may not identify all PDs. However, it should identify important PDs. Risk assessment activities identify critical data and 


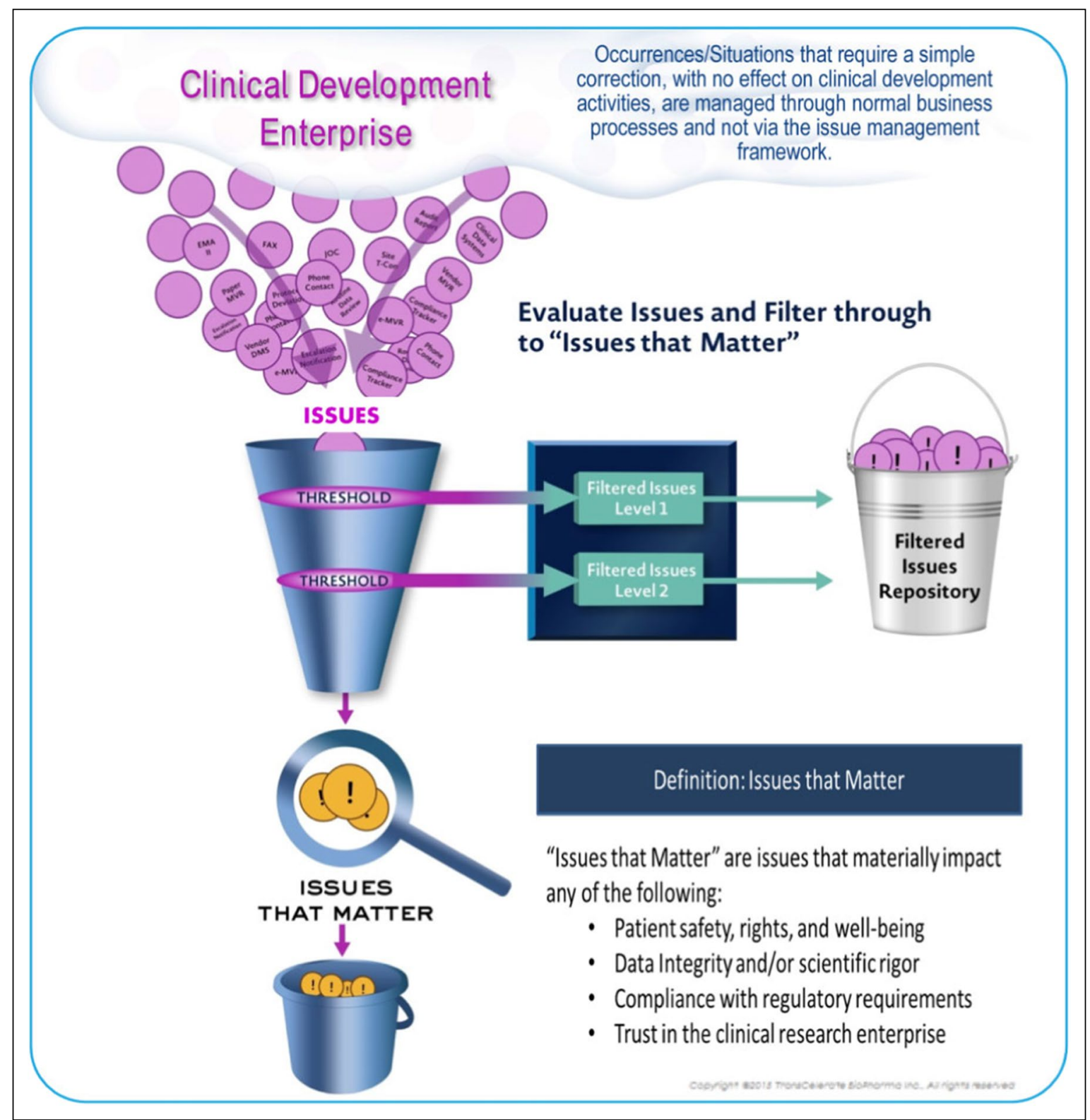

Fig. 5 Issue management illustration

processes that matter most in the clinical study. Armed with this prioritized information, the study team can focus activities on PDs with the greatest impact on participant safety and reliability of study data.

If important PDs are identified during study conduct, which are not included in the PDAP, the study team should consider updating the PDAP.

\section{Classify and Categorize}

Each PD should be classified and categorized upon identification, ideally by the identifier.
- Classify is defined as determining if the PD is important or non-important.

- Categorize is defined as the type of PD (e.g., inclusion/ exclusion).

The classification of a non-important deviation can change. Triggers for reclassification may include:

- meeting a pre-determined threshold

- increase in volume, frequency or cadence

Conversely, PDs classified as important may be reclassified to non-important. 
The PDAP template contains four categories from ICH E3 plus three additional recommended categories. Table 1 in Appendix contains examples of classification and categorization to guide in identifying situations which are important, non-important and not a PD.

\section{Confirm}

PD classification and categorization should be confirmed. It is recommended that an independent study team member (or group), other than the identifier, perform this review. Feedback and possible retraining should be provided to the identifier if the PD is misclassified, miscategorized, or otherwise erroneously reported.

Based on collection method (programmatic and/or manual), important PDs should be reconciled, and all discrepancies should be addressed. Reconciliation may include:

- removal of duplicate PDs (e.g., those identified via both programmatic and manual methods)

- consistency between data point(s) and PD (e.g., transcription error corrected eliminating the PD)

For non-important PDs, periodic aggregate reviews should be completed to identify trends or systemic errors which may meet a threshold to upgrade the classification to important.

\section{Store}

PDs, including the classification and categorization and any associated data points, should be stored in a validated repository or system to support review and reporting (e.g., Clinical Trial Management System [CTMS], Electronic Data Capture [EDC], Trial Master File [TMF] or a custom system).

Key elements for storage considerations are the ability to retrieve or regenerate both important and non-important PDs for various analyses and reporting needs.

The PDAP should be stored with other protocol related decision-making records.

\section{Review and Analyze}

Study teams typically conduct periodic reviews, monitor clinical study data and conduct analyses during study conduct. The results may be leveraged to identify important PDs not previously included in the PDAP and to assess whether frequency or volume of non-important PDs should trigger reclassification. If existing activities cannot be leveraged, additional efforts should be considered.

Important PDs are one of several factors used to determine which participants are excluded from the "per protocol" analysis study population pre-specified in the Statistical Analysis Plan (SAP). Impact of important PDs should be discussed in the CSR.

\section{Report}

Periodic reporting during the clinical study to a central IRB, local IRBs, EC, and/or Health Authorities varies on cadence and content. Both important and non-important PDs should be reported as required.

Guidance for discussing important PDs is addressed in ICH E3. The below recommendations apply to Section 10.2 for both interim and final CSRs.

- Include a high-level, study-specific summary of important PDs that occurred. Discuss impact on participant safety or interpretation of study results. The impact may be by participant level, investigational site level or overall.

- Display number of participants whose important PD(s) resulted in exclusion of any/all of their data from any/all analyses.

- Summarize important PDs by category.

GCP issues are usually described in a separate section of the CSR. However, Section 10.2 may include a reference to those participants whose important PDs resulted from a GCP issue(s).

\section{Close Out}

Important PDs are summarized and included in the CSR and archived. Non-important PDs should be archived in a validated repository or system to support future reviews and PD data sets (e.g., SDTM). Additionally, protocol level important PDs may be considered for use at intermediate or organizational levels. 


\section{Conclusion}

Stakeholders, including sites and CROs, reported frustration, inefficiencies and challenges in multiple areas related to identification and processing of important PDs that could lead to missed issues and create potential patient safety concerns. The variety of definition interpretations was identified as a common factor. Key principles were developed to clarify what constitutes a PD and risk-based approaches from ICH E6 R2 were applied to the definition of an important PD.

A toolkit was developed to support a holistic approach in management of PDs. The tools are flexible to suit a wide variety of indications, study designs, and investigational agents while also providing tools for consistent application within a study, program or organization.

Together, building on the definition of an important PD and using fit-for-purpose tools will support stakeholders for their role in rapid identification and management of important PDs which have the most impact on patient safety or data integrity.

\section{Supplemental Resources}

Toolkit components are available for download from TransCelerate's website [16]. Resources for Common Protocol Templates [17], Quality Management Systems [18] and Risk Based Monitoring [19] are also available.

\section{Author Contributions}

All authors were involved in the writing and editing of this article. The authors gratefully acknowledge the support of TransCelerate BioPharma Inc., a non-profit organization dedicated to improving the health of people around the world by accelerating and simplifying the research and development (R\&D) of innovative new therapies. The authors also gratefully acknowledge the support of the entire TransCelerate Protocol Deviation Team who were essential in development of concepts and tools described in this manuscript.

\section{Funding}

No funding sources were provided to the authors.

\section{Declarations}

\section{Conflict of interest}

The authors declare no conflicts of interest. However, all authors are employees and/or stockholders of the companies with which they are affiliated.

\section{Appendix}




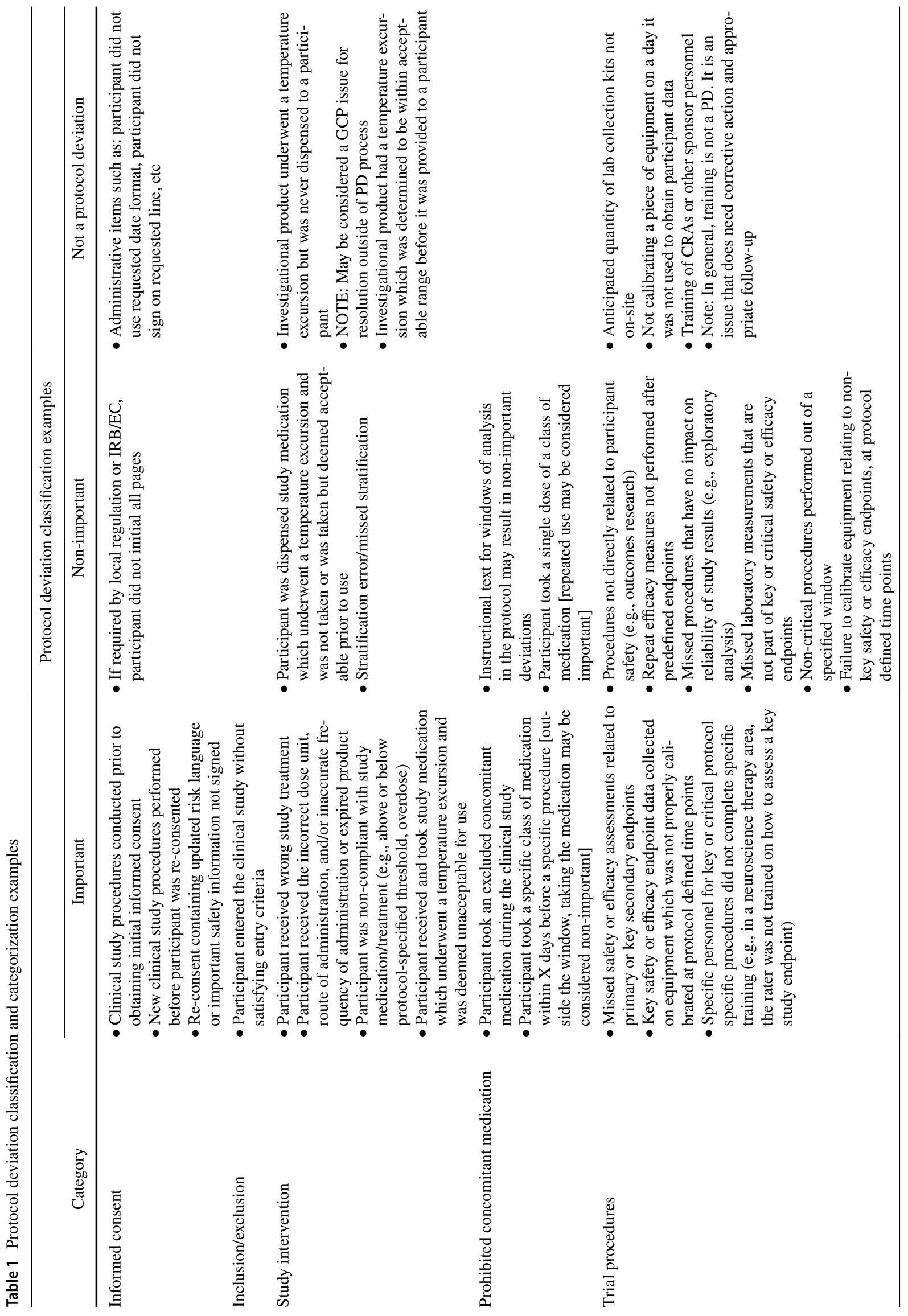




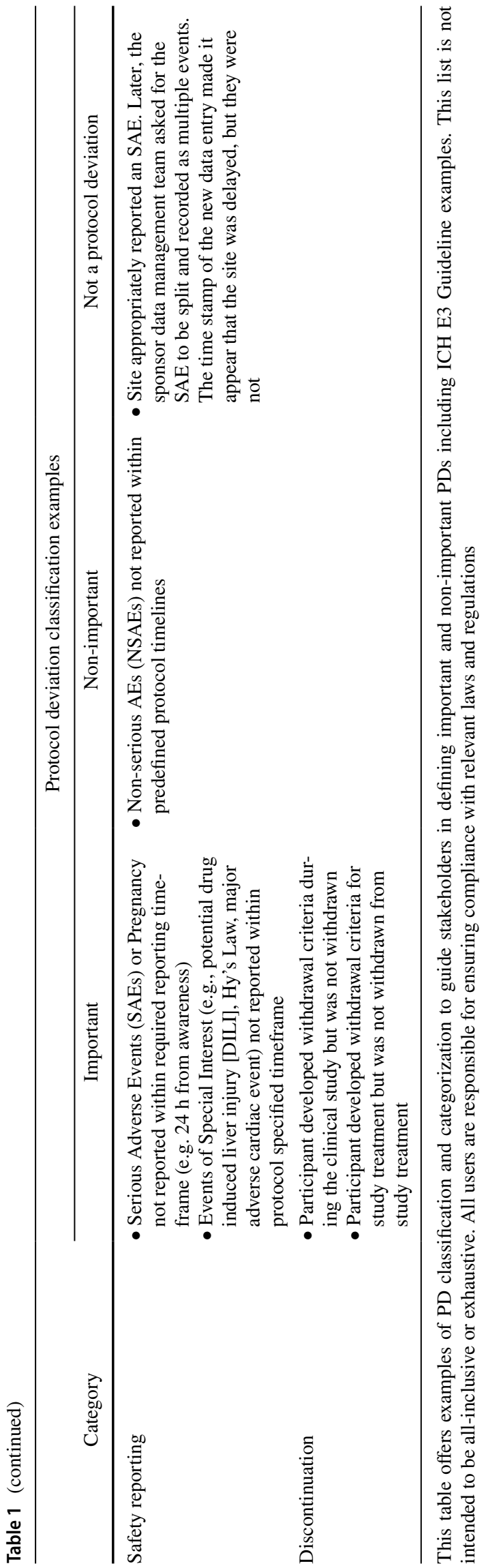

\section{Open Access}

This article is licensed under a Creative Commons Attribution 4.0 International License, which permits use, sharing, adaptation, distribution and reproduction in any medium or format, as long as you give appropriate credit to the original author(s) and the source, provide a link to the Creative Commons licence, and indicate if changes were made. The images or other third party material in this article are included in the article's Creative Commons licence, unless indicated otherwise in a credit line to the material. If material is not included in the article's Creative Commons licence and your intended use is not permitted by statutory regulation or exceeds the permitted use, you will need to obtain permission directly from the copyright holder. To view a copy of this licence, visit http://creativecommons.org/licenses/by/4.0/.

\section{References}

1. ICH GCP. http://www.ema.europa.eu/ema/index.jsp?curl=pages/ regulation/general/general_content_000072.jsp\&mid=WC0b0 $1 \mathrm{ac} 05800268 \mathrm{ad}$.

2. ICH E8. General Considerations for Clinical Trials section 2.2. March 1998

3. ICH E3. Structure and content of clinical study reports section 10.2. July 1996.

4. ICH E3. Guideline: Structure and content of Clinical Study Reports Questions \& Answers (R1). July 2012 question 7. https:// www.ema.europa.eu/en/documents/scientific-guideline/internatio nal-conference-harmonisation-technical-requirements-registrati on-pharmaceuticals-human-use_en-5.pdf.

5. TransCelerate survey results.

6. TransCelerate supplemental site survey results.

7. Galuchie L. Key principles for protocol deviations. Presentation at SCOPE, Orlando, FL, USA, February 2019.

8. Stewart C. Key principles for protocol deviations. Presentation at Clinical Trials Europe, Barcelona, Spain, September 2019.

9. Stewart C. Key principles for protocol deviations. Presentation at SCOPE, Barcelona, Spain, November 2019.

10. Stewart C. Protocol Deviation Management Toolkit: Seeking Public Feedback, TransCelerate public webinar. 22 Oct 2019.

11. Galuchie L. Protocol Deviation Management Toolkit: Seeking Public Feedback, TransCelerate public webinar. 23 Oct 2019.

12. Callery-Damico S, Sam L, Grey T, Greenwood D. TransCelerate's clinical quality management system: issue management. Therap Innov Regul Sci. 2016;50(5):530-5.

13. Meeker-O'Connell A, Sam L, Bergamo N, Little J. TransCelerate's clinical quality management system: from a vision to a conceptual framework. Therap Innov Regul Sci. 2016;50(4):397-413.

14. FDA Guidance for Industry. Oversight of clinical investigationsa risk-based approach to monitoring. August 2013. https://www. fda.gov/media/116754/download.

15. Suprin M, Chow A, Pillwein M, Rowe J, Ryan M, RygielZbikowska B, et al. Quality risk management framework: guidance for successful implementation of risk management in clinical development. Therap Innov Regul Sci. 2019;53(1):36-44.

16. TransCelerate Protocol Deviation Assets: https://www.transceler atebiopharmainc.com/initiatives/protocol-deviations/.

17. TransCelerate Clinical Content \& Reuse: https://www.transceler atebiopharmainc.com/initiatives/clinical-content-reuse/.

18. TransCelerate Quality Management System: https://www. transceleratebiopharmainc.com/initiatives/quality-manag ement-system/.

19. TransCelerate Risk Based Monitoring: https://www.transceler atebiopharmainc.com/initiatives/risk-based-monitoring/. 